Herz 2022 - 47:118-122

https://doi.org/10.1007/s00059-022-05101-1

Accepted: 2 February 2022

Published online: 8 March 2022

(c) The Author(s), under exclusive licence to Springer Medizin Verlag GmbH, ein Teil von Springer Nature 2022

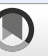

\title{
Catheter ablation for atrial fibrillation: impact on mortality, morbidity, quality of life, and implications for the future
}

\author{
Patrick Dilk ${ }^{1,2} \cdot$ Rolf Wachter $^{1} \cdot$ Gerhard Hindricks ${ }^{2}$ \\ 'Clinic and Policlinic for Cardiology, University Hospital Leipzig, Leipzig, Germany \\ ${ }^{2}$ Department of Electrophysiology, Heart Centre Leipzig, Leipzig, Germany
}

\section{Abstract}

Despite the advances in technologies and techniques in the field of catheter ablation for cardiac arrhythmias, it is estimated that the prevalence of atrial fibrillation (AF) will further increase in the nearest future. The latest trials have proven the beneficial effect on mortality after pulmonary vein isolation in patients with impaired left ventricular function, while no such effect has been seen in patients without left ventricular dysfunction. This raises the question of whether catheter ablation for AF is still suited for the latter patient cohort or whether the endpoint of mortality is not adequate enough. Not only does pulmonary vein isolation reduce the burden of atrial fibrillation, but it also somehow alters the patients' perception of it in the case of recurrence. Independent of the presence of ventricular dysfunction, patients experience a relief of AF-related symptoms, which is accompanied by an increase in quality of life based on the available patient-reported outcome measures, despite AF recurrence. Trials that are currently recruiting patients seek to unveil the accountable circumstances for these remaining uncertainties and help expand our understanding of a procedure that has been routinely performed for two decades.

\section{Keywords}

Arrhythmia $\cdot$ Pulmonary vein isolation $\cdot$ Heart failure $\cdot$ Health-related quality of life $\cdot$ Treatment

The prevalence of atrial fibrillation is expected to rise in the future with our aging population [1]. In the past two decades, pulmonary vein isolation has become the most widely used interventional treatment option for patients with atrial fibrillation. Today, 24,000 catheter ablation procedures are performed per year in Germany [2] and it is likely that these numbers will increase in the future. We aimed to revisit the contemporary scientific evidence for the most relevant aspects of pulmonary vein isolation and assess the future of this procedure.
Origins of pulmonary vein isolation and impact on current treatment strategies

Haïssaguerre et al. first discovered, in the late 1990s, that bursts of ectopy within the pulmonary veins lead to the development of atrial fibrillation [3]. Hence, the initial treatment approach was the elimination of these ectopic foci with catheter ablation. Major limitations of this approach were the high prevalence of pulmonary vein stenosis and multiple origins of pulmonary vein ectopy. These disadvantages spurred further improvements in the procedure. Targeting the conjunction of the pulmonary vein and the left atrial musculature led to the development of pulmonary vein isolation, whereby the ectopic foci within a pulmonary vein were isolated by 
scar tissue, which is created by catheter ablation, located around the pulmonary vein ostium. Further understanding of the pathogenesis of atrial cardiomyopathy and the development of atrial fibrillation contributed to the establishment of complementary ablation techniques such as ablation of complex fractionated electrograms or substrate modification with additional linear ablation lesions.

These technical improvements were accompanied by emerging technological advances, such as the use of high-power short-duration protocols (HPSD), which enable a reduction in the total procedure time, while maintaining comparable recurrence and complication rates [4, 5]. Recent developments, in particular pulsed field ablation, which does not use thermal energy sources, appear to be promising, offering further reductions in procedure time and complication rates [6].

In spite of these technological advances, trials concerning hard clinical endpoints were missing for many years. It took almost 20 years after the original publication by Haïssaguerre et al. to address this lack of clinical evidence. In 2018, the Catheter Ablation Versus Standard Conventional Therapy in Patients with Left Ventricular Dysfunction and Atrial Fibrillation (CASTLE-AF) trial revealed a significant reduction in mortality rates for patients who underwent pulmonary vein isolation for atrial fibrillation compared to medical therapy including rhythm and rate control therapy [7]. The results of a currently published neural network-based analysis of 15,659 patients with heart failure and a mean left ventricular ejection fraction of $27 \%$ reported that the use of beta-blocker medication in the majority of patients with atrial fibrillation does not reduce mortality when compared to patients with sinus rhythm, suggesting that, to date, catheter ablation remains the only mortality-reducing treatment in this very common group of patients ( 0 Fig. 1 ; [8]).

By contrast, the Catheter Ablation Versus Antiarrhythmic Drug Therapy for Atrial Fibrillation (CABANA) trial, which compared catheter ablation for atrial fibrillation with medical therapy, irrespective of the patients' left ventricular function, failed to show a significant difference regarding heart failure hospitalizations, all-cause mortality, or stroke based on an intention-to-treat analysis $[9,10]$. A recently published post hoc analysis of the CABANA trial including patients with heart failure symptoms at enrolment (defined as New York Heart Association class > II) described a comparable effect regarding heart failure hospitalizations and mortality as the CASTLE-AF trial. The authors additionally observed an ameliorated quality of life (QoL) in patients who underwent pulmonary vein isolation based on patient-reported outcome measures (Atrial Fibrillation Effect on Quality of Life [AFEQT] score) when compared with those who received medical treatment [11].

On the basis of these pivotal trials, guidelines recommend catheter ablation for atrial fibrillation to reduce heart failure hospitalization and mortality in patients with impaired left ventricular function or tachycardia-induced cardiomyopathy irrespective of their symptom status; however, the rhythm control strategy for patients who are not affected by heart failure symptoms or impaired left ventricular function is routine and primarily determined by individual patient preference [12].

Another pivotal implication in the treatment of atrial fibrillation with a rhythm control strategy was determined by the Early Treatment of Atrial Fibrillation for Stroke Prevention (EAST AFNET 4) trial, which revealed a significant reduction in adverse cardiovascular events (defined as death from cardiovascular cause, stroke, or heart failure hospitalization), when applying early rhythm control treatment (medical therapy and pulmonary vein isolation), as compared with usual care [13]. The trial was stopped prematurely for effectiveness after a mean follow-up of 5 years. One of the limitations of the trial was seen in the low event rate in the intervention and control arms (3.9 per 100 person-years vs. 5 per 100 person-years; [14]); however, contemporary work highlights the effectiveness of an integrated treatment approach, incorporating not only rhythm, -or rate control, but also adequate anticoagulation to prevent thromboembolic events. The aforementioned effect of early rhythm control therapy was even more ameliorated in a subset of patients with reduced ejection fraction (5.7 vs. 7.9 per 100 person-years), in agreement with the results of the CASTLE-AF [7] and sub-analysis of the CABANA trial [11].

It is noteworthy that mortality rates are highest in wealthier countries (measured by the gross domestic product being above the European average, e.g., Sweden and Germany), compared with poorer countries, despite higher healthcare expenditure per capita. This counterintuitive effect might be the result of a higher prevalence of risk factors, as well as comorbidities, which is far higher in wealthier countries. The authors hypothesize that this effect can also be attributed to a survivor effect and lower detection rate caused by limited access to diagnostic measurements, e.g., ECG [15].

\section{Complications of pulmonary vein isolation and impact on atrial fibrillation-related comorbidities}

Pulmonary vein isolation is considered to be a relatively safe procedure with an overall complication rate of approximately $10 \%$, independent of the technique applied, with minor complications such as vascular injury and pericarditis being the most common. Lethal or permanent compromising complications occur in less than $1 \%$ of procedures [5]. Since the early days of catheter ablation for atrial fibrillation, pulmonary vein stenosis has been one of the most serious complications. Fortunately, the rate of this complication has become negligible, due to improvements in ablation techniques, avoiding direct ablation within the non-muscular aspects of the pulmonary veins and instead focusing on the adjacent atrial aspects [16]. Periprocedural stroke is considered to be another feared major complication of catheter ablation, also occurring in less than $1 \%$ of procedures, with increased rates when anticoagulation is discontinued [17]. Although catheter ablation for atrial fibrillation has led to a significant decrease in atrial fibrillation-related symptoms, mainly due to preservation of sinus rhythm, it seems somewhat discouraging that current data could not demonstrate a clinically impactful reduction in the rate of stroke [18]. This could be attributed to the significant effectiveness of continuation of oral anticoagulation as well as the residual stroke rate being ascribed to non-atrial fibrillation-re- 


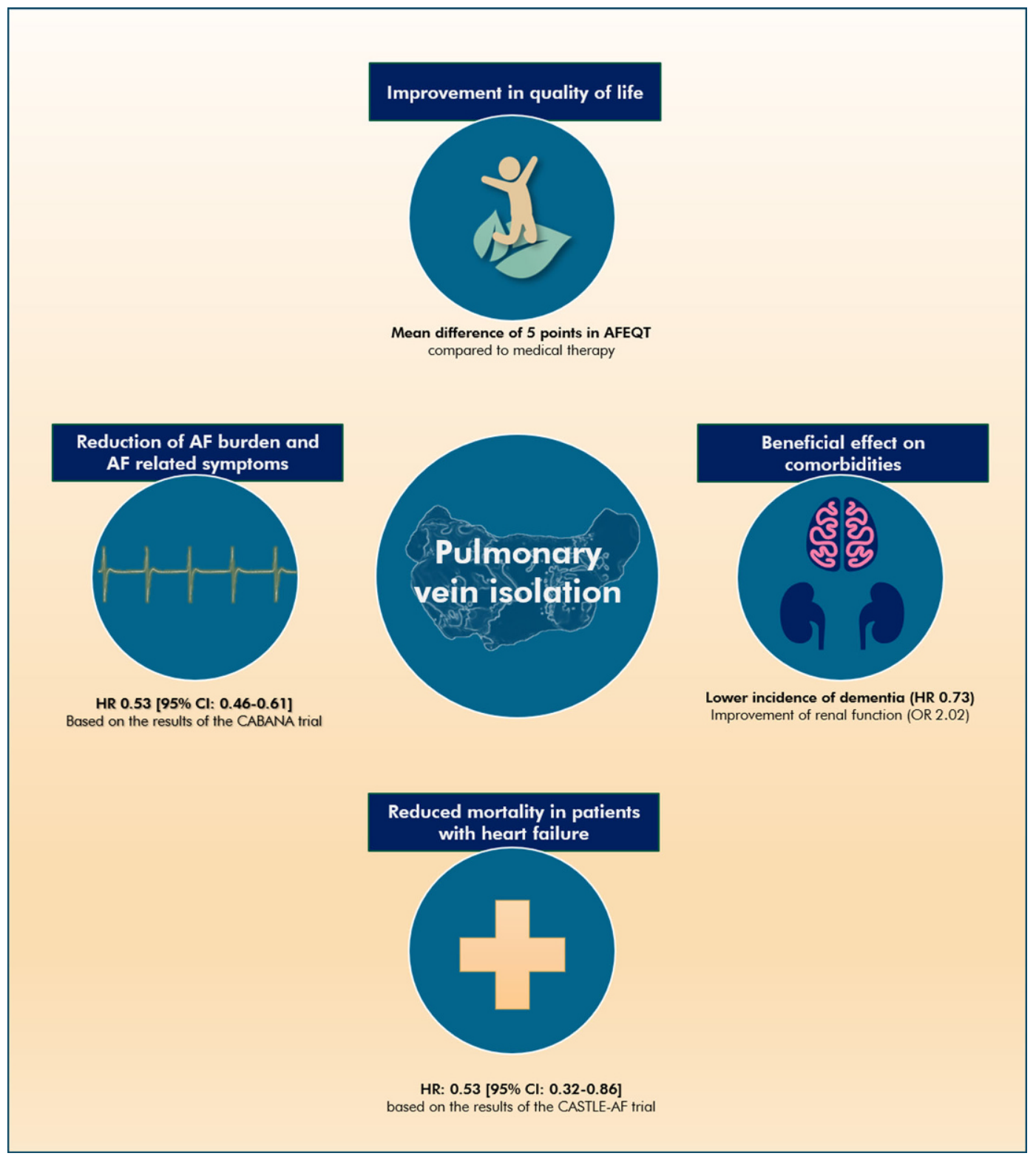

Fig. $1<$ Advantages of pulmonary vein isolation as interventional treatment option for patients with atrial fibrillation (AF). AFEQT Atrial Fibrillation Effect on Quality of Life questionnaire, $C A$ BANA Catheter Ablation Versus Antiarrhythmic Drug Therapy for Atrial Fibrillation trial, CASTLEAF Catheter Ablation Versus Standard Conventional Therapy in Patients with Left Ventricular Dysfunction and Atrial Fibrillation trial, Cl confidence interval, $H R$ hazard ratio, $O R$ odds ratio

lated thromboembolism due to atrial cardiomyopathy or non-cardioembolic stroke [18]. The results of the ongoing OCEANAF trial will provide further evidence regarding the latter [19].

In addition to an increased risk for thromboembolic events, atrial fibrillation is associated with further cardiac comorbidities. With highly preserved atrioventricular conduction properties, atrial fibrillation results in high ventricular beating frequencies, which are, in conjunction with asynchronous ventricular contractions, able to mediate a deterioration of ventricular function [20]. Catheter ablation for atrial fibrillation can reverse the condition and normalize left ventricular function and left ventricular parameters as investigated by cardiac magnet resonance imaging [21]. Currently recruiting trials will investigate whether pulmonary vein isolation alone or in conjunction with other treatment modalities will influence the outcome of other comorbidities such as arterial or pulmonary hypertension ( $\bullet$ Fig. $\mathbf{1}$; $[22,23])$.

Atrial fibrillation not only leads to a deterioration in cardiovascular function, but it also has an impact on multiple systems, including renal, intestinal, or cognitive function. A retrospective analysis of approximately 27,000 patients with atrial fibrillation, who either received medical treatment or catheter ablation revealed that interventional treatment resulted in a significantly lower incidence of dementia, including Alzheimer disease and vascular dementia [24]. Regarding renal function, atrial fibrillation is associated with chronic kidney disease, worsening of creatinine clearance, and vice versa. Thus, the effect of restoring sinus rhythm in these patients with catheter ablation resulted in an improvement in renal function over a 5 -year follow-up, especially in those patients who had no recurrence of atrial fibrillation [25].

Even in conjunction with the current COVID-19 pandemic, atrial fibrillation is associated with a higher rate of mechanical ventilation and an even higher mortality rate. Additionally, hospitalization for 
COVID-19 is associated with a higher risk for new-onset atrial fibrillation [26].

\section{Postinterventional atrial fibrillation burden and impact on quality of life}

The aforementioned trials not only demonstrated a potential beneficial effect on mortality and hard clinical outcomes after pulmonary vein isolation, but also reported a significantly decreased burden of atrial fibrillation, when compared to medical treatment (- Fig. 1). The Medical Antiarrhythmic Treatment or Radiofrequency Ablation in Paroxysmal Atrial Fibrillation (MANTRA-PAF) trial revealed a timedependent effect on atrial fibrillation burden after catheter ablation for paroxysmal atrial fibrillation when compared to antiarrhythmic drug therapy. It failed to prove a difference in atrial fibrillation recurrence after pulmonary vein isolation compared to medical therapy in the first 18 months of follow-up [27]. At 24 months, atrial fibrillation burden was significantly lower in the ablation group. This effect persisted throughout a 5-year follow-up [28]. Post hoc analysis of the CABANA and CASTLEAF trial also demonstrated a significant lower burden of atrial fibrillation in patients who received catheter ablation compared to medical therapy, which was associated with fewer cases of heart failure hospitalizations $[29,30]$. Additionally, these post hoc analyses investigated the effect of pulmonary vein isolation on patient-reported outcomes, measured by established QoL questionnaires.

Quality of life in atrial fibrillation is usually measured by the disease-specific AFEQT questionnaire. Scores range between 0 and 100, with higher scores indicating better QoL. The beneficial effect of treatment regarding change in sum score was greater in the catheter ablation group with a mean difference of 5 points, compared with the antiarrhythmic drug treatment group [31]. While this favorable effect persisted throughout a 5-year followup when assessing QoL with the AFEQT, there has been a decline when different questionnaires, which are not as specific for atrial fibrillation symptom burden (short form 36; SF36), are selected [32]. It is not surprising that the total atrial fibrillation burden correlates indirectly with patients' daily activity level after pulmonary vein isolation, which may contribute to a lower sum score in patient-reported outcome measures (• Fig. 1; [33]).

Intriguingly, a prospective trial of 118 patients who were assigned for pulmonary vein isolation and who underwent intensified rhythm monitoring before and after catheter ablation revealed a significant increase in asymptomatic atrial fibrillation episodes after pulmonary vein isolation [34]. Yet it is unclear whether this effect, alongside the aforementioned improvement in QoL sum scores, can be attributed to: (1) changes in the autonomous tone after pulmonary vein isolation, caused by an additional ablative effect on the ganglionated plexi, which are directly adjacent to the pulmonary vein insertion [35]; (2) changes in the arrhythmia pattern or substrate; or (3) a possible placebo effect. The latter will be assessed in a currently recruiting randomized, double-blind, shamcontrolled trial (NCT05119231), which primarily addresses the changes in QoL after pulmonary vein isolation in patients without impaired left ventricular function [36]. This trial will make it possible to estimate the "true" effect of pulmonary vein isolation on QoL and also quantify the placebo effect of pulmonary vein isolation.

\section{Conclusion}

The latest trials on atrial fibrillation have reported the beneficial effect on mortality after pulmonary vein isolation in patients with impaired left ventricular function, while no such effect has been seen in patients without left ventricular dysfunction. This raises the question of whether catheter ablation for atrial fibrillation is still suited for the latter patient cohort or whether the endpoint of mortality is not adequate. Independent of the presence of ventricular dysfunction, patients experience a relief of atrial fibrillationrelated symptoms after pulmonary vein isolation, which is accompanied by an increase in quality of life. Trials that are currently recruiting patients seek to unveil the accountable circumstances for these remaining uncertainties and expand our knowledge of a procedure that has been routinely performed for two decades.

\section{Corresponding address}

\section{Dr. Patrick Dilk}

Clinic and Policlinic for Cardiology, University Hospital Leipzig

Strümpellstr. 39, 04289 Leipzig, Germany

patrick.dilk@helios-gesundheit.de

\section{Declarations}

Conflict of interest. P. Dilk, R. Wachter, and G. Hindricks declare that they have no competing interests.

For this article no studies with human participants or animals were performed by any of the authors. All studies performed were in accordance with the ethical standards indicated in each case.

\section{References}

1. Kornej J, Börschel CS et al (2020) Epidemiology of atrialfibrillation in the 21stcentury: novelmethods and new insights. Circ Res 127:4-20. https://doi. org/10.1161/CIRCRESAHA.120.316340

2. Raatikainen MJP, Arnar DO et al (2017) A decade of information on the use of cardiac Implantable electronic devices and interventional electrophysiological procedures in the European Society of Cardiology countries: 2017 report from the European Heart Rhythm Association. Europace 19:ii1-ii90. https://doi.org/10.1093/europace/ eux258

3. Haïssaguerre M, Jaïs P et al (1998) Spontaneous initiation of atrial fibrillation by ectopic beats originating in the pulmonary veins. $\mathrm{N}$ Engl J Med 339:659-666. https://doi.org/10.1056/ NEJM199809033391003

4. Kottmaier M, Popa M et al (2020) Safety and outcome of very high-power short-duration ablation using $70 \mathrm{~W}$ for pulmonary vein isolation in patients with paroxysmal atrial fibrillation. Europace 22:388-393. https://doi.org/10.1093/ europace/euz342

5. de Greef Y, Ströker E et al (2018) Complications of pulmonary vein isolation in atrial fibrillation: predictors and comparison between four different ablation techniques: results from the Mlddelheim PVI-registry. Europace 20:1279-1286. https://doi. org/10.1093/europace/eux233

6. Reddy VY, Neuzil P et al (2019) Pulsed field ablation for pulmonary vein isolation in atrial fibrillation. J Am Coll Cardiol 74:315-326. https://doi.org/10. 1016/j.jacc.2019.04.021

7. Marrouche NF, Brachmann J et al (2018) Catheter ablation for atrial fibrillation with heart failure. N Engl J Med 378:417-427. https://doi.org/10. 1056/NEJMoa1707855

8. Karwath A, Bunting KV et al (2021) Redefining $\beta$-blocker response in heart failure patients with sinus rhythm and atrial fibrillation: a machine learning cluster analysis. Lancet 398:1427-1435. https://doi.org/10.1016/S0140-6736(21)01638-X

9. Mark DB, Anstrom KJ et al (2019) Effect of catheter ablation vs medical therapy on quality of life among patients with atrial fibrillation: the CABANA randomized clinical trial. JAMA 321:1275-1285. https://doi.org/10.1001/jama.2019.0692

10. Packer DL, Mark DB et al (2019) Effect of catheter ablation vs antiarrhythmic drug therapy on 
mortality, stroke, bleeding, and cardiac arrest among patients with atrial fibrillation: the CABANA randomized clinical trial. JAMA 321:1261-1274. https://doi.org/10.1001/jama.2019.0693

11. Packer DL, Piccini JP et al (2021) Ablation versus drug therapy for atrial fibrillation in heart failure: results from the CABANA trial. Circulation 143:1377-1390. https://doi.org/10. 1161/CIRCULATIONAHA.120.050991

12. Hindricks G, Potpara T et al (2021) 2020 ESC Guidelines for the diagnosis and management of atrial fibrillation developed in collaboration with the European Association for Cardio-Thoracic Surgery (EACTS): The Task Force for the diagnosis and management of atrial fibrillation of the European Society of Cardiology (ESC) Developed with the special contribution of the European Heart Rhythm Association (EHRA) of the ESC. Eur Heart J 42:373-498. https://doi.org/10.1093/ eurheartj/ehaa612

13. Kirchhof $P$, Camm AJ et al (2020) Early rhythmcontrol therapy in patients with atrial fibrillation. N Engl J Med 383:1305-1316. https://doi.org/10. 1056/NEJMoa2019422

14. Bunch TJ, Steinberg BA (2020) Revisiting rate versus rhythm control in atrial fibrillation - timing matters. N Engl J Med 383:1383-1384. https://doi. org/10.1056/NEJMe2027180

15. Al-Khayatt BM, Salciccioli JD et al (2021) Paradoxical impact of socioeconomic factors on outcome of atrial fibrillation in Europe: trends in incidence and mortality from atrial fibrillation. Eur Heart J 42:847-857. https://doi.org/10.1093/eurheartj/ ehaa 1077

16. Narui R, Tokuda Metal (2017) Incidence and factors associated with the occurrence of pulmonary vein narrowing after cryoballoon ablation. Circ Arrhythm Electrophysiol. https://doi.org/10.1161/ CIRCEP. 116.004588

17. Di Biase L, Burkhardt JD et al (2014) Periprocedural stroke and bleeding complications in patients undergoing catheter ablation of atrial fibrillation with different anticoagulation management: results from the Role of Coumadin in Preventing Thromboembolism in Atrial Fibrillation (AF) Patients Undergoing Catheter Ablation (COMPARE) randomized trial. Circulation 129:2638-2644. https://doi.org/10.1161/CIRCULATIONAHA.113. 006426

18. Barra S, Narayanan K et al (2019) Atrial fibrillation ablation and reduction of stroke events: understanding the paradoxical lack of evidence. Stroke 50:2970-2976. https://doi.org/10.1161/ STROKEAHA.119.026890

19. Verma A, Birnie DH and the OCEAN investigators (2014) Optimal anticoagulation for higher risk patients post-catheter ablation for atrial fibrillation trial: NCT02168829, 327494. https://ClinicalTrials. gov/show/NCT02168829

20. Martin CA, Lambiase PD (2017) Pathophysiology, diagnosis and treatment of tachycardiomyopathy. Heart 103:1543-1552. https://doi.org/10.1136/ heartjnl-2016-310391

21. Stegmann C, Jahnke C et al (2021) Cardiovascular magnetic resonance-based predictors of complete left ventricular systolic functional recovery after rhythm restoration in patients with atrial tachyarrhythmia. Europace. https://doi.org/10. 1093/europace/euab174

22. Arif $E$, de Jong $M$ (2014) Treatment of atrial fibrillation in patients by pulmonary vein isolation in combination with renal denervation or pulmonary vein isolation only: NCT02115100, 9206. https:// ClinicalTrials.gov/show/NCT02115100

\section{Katheterablation bei Vorhofflimmern: Einfluss auf Mortalität, Morbidität und Lebensqualität sowie Ausblick in die Zukunft}

Trotz der technologischen und technischen Weiterentwicklung auf dem Gebiet der Katheterablation von Herzrhythmusstörungen ist anzunehmen, dass die Prävalenz von Vorhofflimmern (VHF), als häufigster Arrhythmie, in naher Zukunft weiter zunehmen wird. In jüngsten Studien ließ sich bei Patienten mit eingeschränkter linksventrikulärer Funktion eine positive Wirkung auf die Mortalität nach Pulmonalvenenisolation nachweisen, während bei Patienten ohne linksventrikuläre Dysfunktion ein solcher Effekt nicht zu verzeichnen war. Entsprechend stellt sich hierbei die Frage, ob sich die Katheterablation bei VHF noch für letztgenannte Patientenkohorte eignet und inwieweit der Endpunkt der Mortalität bei diesen Patienten gerechtfertigt ist. Es ist bekannt, dass eine Pulmonalvenenisolation nicht nur die VHF-Last reduziert, sondern auch die individuelle Wahrnehmung im Fall eines Rezidivs verändert. Nach einer Katheterablation zeigen Patienten trotz VHF-Rezidiven eine Linderung der VHFassoziierten Symptomatik, was zusätzlich mit einer Verbesserung der Lebensqualität einhergeht, unabhängig davon, ob eine ventrikuläre Dysfunktion vorliegt. Die Ursachen der veränderten Wahrnehmung des VHF nach einer Katheerablation sind derzeit noch unbekannt und werden in aktuell rekrutierenden Studien untersucht. Die Ergebnisse dieser Untersuchungen werden dazu beitragen, das Verständnis einer Prozedur, welche seit 2 Jahrzehnten routinemäßig durchgeführt wird, weiter aufzuklären.

\section{Schlüsselwörter}

Arrhythmie · Pulmonalvenenisolation · Herzinsuffizienz · Gesundheitsbezogene Lebensqualität . Behandlung

23. Havranek S (2019) Catheter ablation of atrial fibrillation in patients with pulmonary hypertension hypertension: a randomised study: NCT04053361 GH-18-02-00027-0-202. https://ClinicalTrials.gov/ show/NCT04053361

24. Kim D, Yang P-S et al (2020) Less dementia after catheterablation for atrial fibrillation: a nationwide cohort study. Eur Heart J 41:4483-4493. https:// doi.org/10.1093/eurheartj/ehaa726

25. Park J-W, Yang P-S et al (2019) Five-year change in the renal function after catheter ablation of atrial fibrillation. JAm Heart Assoc 8:e13204. https://doi. org/10.1161/JAHA.119.013204

26. Mountantonakis SE, Saleh M et al (2021) Atrial fibrillation is an independent predictor for inhospital mortality in patients admitted with SARSCoV-2 infection. Heart Rhythm 18:501-507. https://doi.org/10.1016/j.hrthm.2021.01.018

27. Raatikainen MJP, Hakalahti A et al (2015) Radiofrequency catheter ablation maintains its efficacy better than antiarrhythmic medication in patients with paroxysmal atrial fibrillation: Ontreatment analysis of the randomized controlled MANTRA-PAF trial. Int J Cardiol 198:108-114. https://doi.org/10.1016/j.ijcard.2015.06.160

28. Nielsen JC, Johannessen A et al (2017) Long-term efficacy of catheter ablation as first-line therapy for paroxysmal atrial fibrillation: 5-year outcome in a randomised clinical trial. Heart 103:368-376. https://doi.org/10.1136/heartjnl-2016-309781

29. Poole JE, Bahnson TD et al (2020) Recurrence of atrial fibrillation after catheter ablation or antiarrhythmic drug therapy in the CABANA trial. J Am Coll Cardiol 75:3105-3118. https://doi.org/ 10.1016/j.jacc.2020.04.065

30. Brachmann J, Sohns Cet al (2021) Atrial fibrillation burden and clinical outcomes in heart failure: the CASTLE-AF trial. JACC Clin Electrophysiol 7:594-603. https://doi.org/10.1016/j.jacep.2020. 11.021
31. Kotecha D, Ahmed A et al (2016) Patient-reported outcomes for quality of life assessment in atrial fibrillation: a systematic review of measurement properties. PLoSONE 11:e165790. https://doi.org/ 10.1371/journal.pone.0165790

32. Siontis KC, loannidis JPA et al (2016) Radiofrequency ablation versus antiarrhythmic drug therapy for atrial fibrillation: meta-analysis of quality of life, morbidity, and mortality. JACC Clin Electrophysiol 2:170-180. https://doi.org/10. 1016/j.jacep.2015.10.003

33. Proietti R, Birnie D et al (2018) Postablation atrial fibrillation burden and patient activity level: insights from the DISCERN AF study. J Am Heart Assoc 7:e10256. https://doi.org/10.1161/JAHA. 118.010256

34. Hindricks G, Piorkowski Cet al (2005) Perception of atrial fibrillation before and after radiofrequency catheter ablation: relevance of asymptomatic arrhythmia recurrence. Circulation 112:307-313. https://doi.org/10.1161/CIRCULATIONAHA.104. 518837

35. Stavrakis S, Nakagawa Het al (2015) The role of the autonomic ganglia in atrial fibrillation. JACC Clin Electrophysiol 1:1-13. https://doi.org/10.1016/j. jacep.2015.01.005

36. Wachter R, Dagres N, the PVI-SHAM-AF Study group (2021) Pulmonary Vein Isolation vs SHAMpulmonary Vein Isolation for Symptomatic Relief in Patients With AF-a Randomised Trial: NCT05119231, PVI-SHAM-AF CIV-21-01-035584. https://ClinicalTrials.gov/show/NCT05119231 